\title{
Puentes, sociedad e ingeniería
}

\section{Bridges, society and engineering}

\author{
$\underline{\text { P. Plasencia-Lozano }}^{(*)}$
}

\section{RESUMEN}

El presente texto reflexiona sobre el desapego de la sociedad hacia la estética de las obras civiles ubicadas en el territorio. Tomando los puentes como ejemplo, se analiza la visión que de ellos tienen los propios ingenieros; a continuación se relacionan los principales parámetros que rigen su concepción y se evalúa la importancia de la componente paisajística del proyecto, advirtiéndose una divergencia de opiniones entre unos autores y otros. La existencia de cuatro vías de aproximación social hacia el elemento constructivo «puente»-descritas asimismo en el texto- demuestran tanto la fuerte presencia de éste en la cultura como su potencia en tanto que elemento paisajístico. El último apartado del texto sintetiza las conclusiones resultantes de la reflexión.

Palabras clave: Puentes; paisaje; estética; ingeniería estructural; diseño conceptual; patrimonio.

\section{ABSTRACT}

The article focuses on the disaffection for the aesthetics of civil engineering. Taking the case of bridges as example, the text includes opinions from different stakeholders, such as bridge designers, researchers and university professors; moreover, key parameters for the conception of a bridge project are described, and the importance of the landscape parameter is considered. Furthermore, it explain the existence of four approximation routes to bridges and society which demonstrates the importance of bridges in our culture and its potential relevance as landscape marks. The last section summarizes the conclusions.

Keywords: Bridges; landscape; aesthetic; structural engineering; conceptual design; cultural heritage.

(*) Ingeniero consultor. Cáceres (España).

Persona de contacto/Corresponding author: pedroplasencia@gmail.com (P. Plasencia-Lozano)

Cómo citar este artículo/Citation: Plasencia-Lozano, P. (2014). Puentes, sociedad e ingeniería. Informes de la Construcción, 66(535): eo32, doi: http://dx.doi.org/10.3989/ic.13.041.

Licencia/License: Salvo indicación contraria, todos los contenidos de la edición electrónica de Informes de la Construcción se distribuyen bajo una licencia de uso y distribución Creative Commons Reconocimiento no Comercial 3.o. España (cc-by-nc). 


\section{INTRODUCCIÓN}

El objeto último de la ingeniería civil radica en la concepción y construcción de elementos que se insertan en el territorio. Dichos elementos, atendiendo a su naturaleza, pueden tener un fuerte impacto visual sobre los usuarios de ese territorio en el presente y en el futuro, condicionando en gran medida la relación de estos observadores con el lugar donde se insertan.

Sin embargo, creemos que la sociedad no aprecia en general las virtudes estéticas de los artefactos ingenieriles -aportaremos datos a lo largo del texto que subrayan esta opinión-; paradójicamente, mientras que éstos modifican en alto grado el territorio, los usuarios visuales de dicho territorio actúan como protagonistas del cuento monterrosiano: «cuando despertó, la carretera todavía estaba allí» (1).

\section{EL PUENTE, VISTO POR LOS INGENIEROS}

Habida cuenta de lo amplio que resulta el universo de la obra pública centraremos este texto en la figura de los puentes, tomándolos como ejemplo de artefacto que el ingeniero dispone sobre el territorio, y cuya utilidad generalmente lo sitúa frente a los ojos de un amplio conjunto poblacional. Pensamos que, en primer lugar, cabe preguntarse qué representa el puente para la propia profesión ingenieril; analizando diversos escritos de proyectistas se observa una recurrencia de éstos por citar el libro escrito por el ingeniero americano David B. Steinman (1886-1960). Dada su influencia, consideramos oportuno reproducir uno de sus más célebres y vibrantes fragmentos:

«Los puentes simbolizan ideales y aspiraciones de la humanidad. Salvan las barreras que nos separan y juntan pueblos, comunidades y naciones en unidades más íntimas. Acortan distancias, aceleran el transporte y facilitan el comercio. Soportan sus cargas para aligerar las tareas de los hombres [...]. Son esfuerzos conjuntos de diseñadores y operarios, de ciencia y destreza. Conforman la visión e iniciativa de las comunidades; son monumentos útiles, dedicados al bienestar de futuras generaciones. Son eslabones vitales en el camino hacia la fraternidad universal del género humano [...] Han inspirado a los poetas a traves de los siglos y han estado en la literatura y la leyenda. Hay algo en los grandes vanos que excita la imaginacion. Desde sus cimientos excavados en la roca hasta sus torres y vanos abovedados, un puente tiene algo de prodigio y de poesia. Es una conjunción mística de resistencia y belleza, una mágica combinación de gracia, encumbradas líneas y desafiante poder» (2).

Esta definición encierra y abarca todas las cualidades que pueden atribuirse a un puente bajo diversas perspectivas: el puente como ideal constructivo, el puente como símbolo de toda una sociedad, el puente como logro tecnológico con el que vencer un obstáculo, el puente como monumento, el puente como superador de fronteras, el puente como herramienta económica y comercial. Y si bien nos maravillamos ante la destreza constructiva de los ingenieros romanos, su conocimiento de la estereotomía o la eficacia de sus diseños -con unas calzadas amplias y sin alomamientos (3)- reconocemos que la historia de la evolución de los puentes en los siglos XVIII, XIX y XX en Inglaterra, América y Francia, es la historia apasionante de la búsqueda de la superación y la concrección del paso de la edad moderna a la contemporánea.
La historia de la construcción, directamente unida tanto a la de la evolución de las herramientas del cálculo (esas mismas que hoy sirven tanto para los avances en ingeniería nuclear, en medicina, en astronáutica o en telecomunicaciones) como a la aparición de nuevos materiales constructivos como el hierro, el acero o el hormigón, nos obliga a distinguir la especial singularidad de los puentes. Como dice el poema de Macaulay, Lays of Ancient Rome, "de todos los inventos, exceptuando solamente el alfabeto y la imprenta, aquellos que abrevian las distancias han sido los que más han hecho por la civilización de nuestra especie» (4). Santiago Hernández Fernández irá más allá al atribuir «alma» a los puentes, «o, al menos, la capacidad de provocar sentimientos en quienes los han construido y en aquellos que, cuando los contemplan, pueden ver a todos quienes han hecho posible que su obra sirva a miles de personas durante siglos. El puente es más que un libro, más que una película, más que un relato, más que una herramienta... el puente nos permite vivir una «experiencia» que nos une a su origen, su pasado, su presente y su futuro» (5).

Seguimos a Manterola cuando escribe que «de todas las obras públicas, las más objetuales, las que más historia y tradición tienen, y a las que con menor dificultad se les atribuye una dimensión estética notable, considerándose en algunos casos como obras de arte, son los puentes». En efecto, quizá los puentes sean las obras públicas más icónicas, pero aun así -vuelve a decir Manterola- «no han entrado en el radio de acción de los críticos de arte. Son como transparentes: no se ven, no interesan ni se entienden» (6), poniendo de manifiesto ese escaso interés social al que aludíamos antes, y que se manifiesta en la escasa atención prestada por las autoridades y operadores turísticos hacia obras como el puente de Pino o de Requejo (José E. Ribera, 1914), que permanece oculto en un rincón de la provincia de Zamora pese a su monumentalidad y su compenetración en el paisaje (Figura 1).

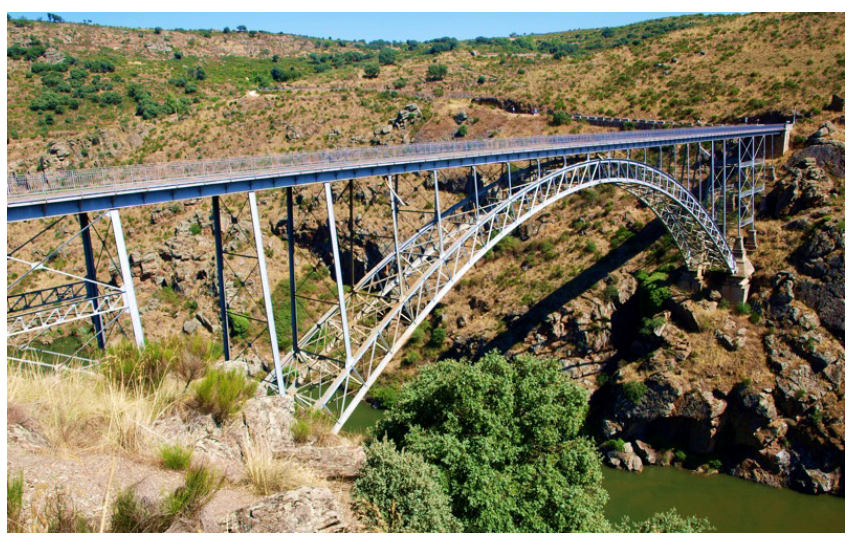

Figura 1. Puente de Pino, en Zamora.

Sin duda, entonces, el puente constituye el icono de la ingeniería civil. Con los puentes el ingeniero se presenta ante el mundo, por los puentes es juzgado y en los puentes encuentra la síntesis de cuantas materias afectan a su profesión. El puente es un problema de cálculo de estructuras porque hay que resolver el equilibrio de un esquema de fuerzas; es un problema de materiales porque hay que dilucidar qué elementos se emplean; es un problema de dinámica porque hay que enfrentarse a las dilataciones y alteraciones generadas por los móviles que lo aprovechan; es un problema geotécnico porque el terreno es la base que soporta los esfuerzos; 
es un problema hidráulico porque en el diseño de sus pilas encontraremos la influencia de las dinámicas fluviales y en el vacío de sus luces la sección necesaria para el paso de un determinado caudal; es un problema de tráfico porque éste incide en la elección de la anchura del tablero; es un problema urbanístico porque habremos de elegir el número de vías, viales o carriles a albergar; es un problema de ferrocarriles o caminos, porque la forma se ve afectada por el trazado y la rasante que debe asumir; es un problema de organización de obra porque la economía de costes y plazos condicionarán el método constructivo -como ocurre en el puente Arcos de Almonte (Cáceres, Siegrist y Moreno, S.L., 2006), cuyas fases constructivas aparecen representadas en la Figura 2 (7)- es un problema legal porque su disposición afecta siempre a terrenos que gozan de figuras de protección o servidumbres; es un problema medioambiental porque su construcción altera fuertemente el medio donde se inserta; es un problema paisajístico porque su estampa permanece construida frente a la vista de usuarios y no usuarios durante varias decenas de años -algunos llegan a pervivir de una Edad a otra-; finalmente, es un problema de administración de caudales públicos, pues el coste de la construcción de infraestructuras es relevante dentro de los presupuestos de cualquier administración.

Por tanto, así como Rossi dice que «el túmulo largo de seis pies y ancho de tres es la arquitectura más intensa y más pura porque se identifica en el hecho» (8), entendemos que eso es el puente para la ingeniería: un símbolo del hacer del ingeniero civil, un «estilete estructural y constructivo» (9) en palabras de Martínez Calzón; «el más antiguo y, a la vez, el más moderno problema de la ingeniería civil, [...] el símbolo más antiguo de la civilización en el paisaje, y [...] causa de legítimo orgullo para el ingeniero» (10), como dice Abercombrie (Figura 3); el «espíritu unificador» común a todos los

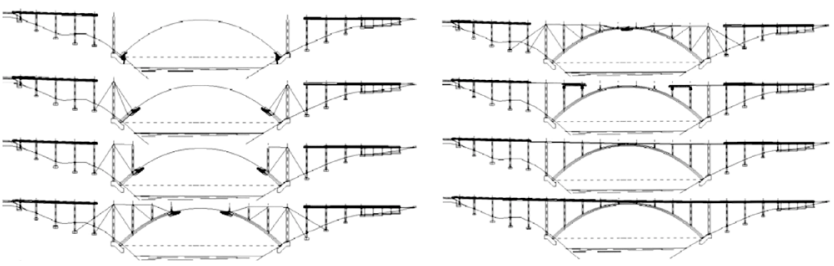

Figura 2. Secuencia constructiva del puente Arcos de Almonte, en Cáceres.

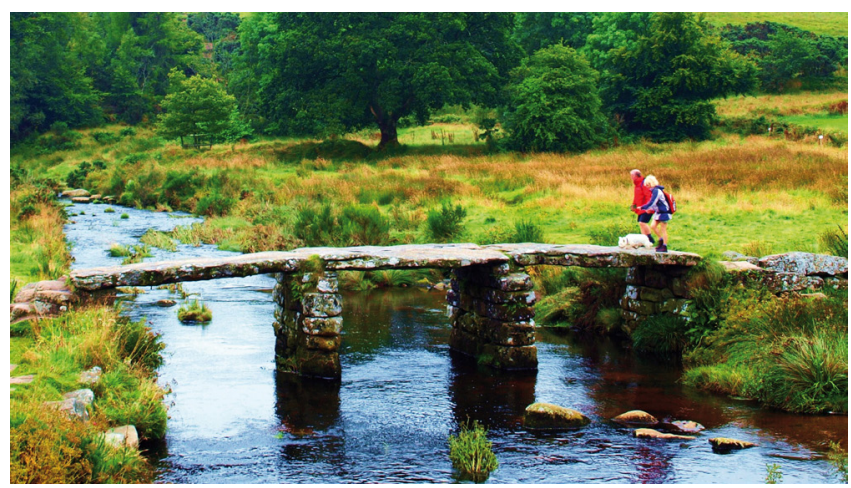

Figura 3. «El más moderno y antiguo problema de la ingeniería civil»: Clapper Bridge, en Postbridge (Reino Unido) ${ }^{1}$. ingenieros, el «espíritu de visión, de invención, de coraje, de sacrificio y de dedicación» (11) de todos ellos, según Steinman; la obra de ingeniería más conocida, que logra «traspasar los ámbitos de la especialización para interesar a un público más amplio» (12), concluye Aguiló. No en vano, la primera exposición dedicada a la ingeniería en un museo se dedicó al ingeniero Robert Maillart y a su puente de Salginatobel (construido en 1930), y tuvo lugar en 1947 en el MoMa de Nueva York (13).

$\mathrm{Al}$ igual que Aguiló, Barker y Puckett opinan que los puentes interesan a amplios colectivos sociales, y así escriben que «los puentes son importantes para todo el mundo. Pero no son vistos del mismo modo por todo el mundo, y precisamente esto es lo que hace su estudio tan fascinante. Un mismo puente sobre un pequeño río será visto de distintas maneras porque los ojos de cada espectador miran lo que es único con respecto a sí mismos. Alguien que viaje sobre el puente cada día mientras va a trabajar se da cuenta de que el puente está allí porque sobre la carretera aparece un guardarraíl. Otros quizá recuerden la época en que el puente fue construido y la distancia tan larga que tenían que recorrer entonces para visitar a los amigos o llevar a los niños al colegio. Los gestores públicos miran el puente como un enlace entre barrios y como camino para dar acceso a la policía y a los bomberos y garantizar la protección de esos barrios, además de permitir el acceso a los hospitales. En el mundo empresarial, el puente es visto como una posibilidad de abrir nuevos mercados y expandir el comercio. Un artista considerará el puente y su entorno como un sujeto posible para un cuadro futuro. Un teólogo quizá lo vea como un símbolo de la relación entre Dios y los hombres. Mientras que el propietario de una barcaza, mirando hacia arriba cuando pase bajo el puente, tendrá una vista completamente diferente. Todo el mundo está mirando el mismo puente, pero éste produce diferentes emociones e imágenes visuales en cada uno de sus observadores» (14).

\section{3. ¿UN OBJETO EN EL TERRITORIO O UN OBJETO ESTRUCTURAL?}

Algunos proyectistas de puentes tienden a jerarquizar y ordenar por importancia relativa el conjunto de parámetros y condicionantes que determinan el diseño final de su obra. Por ejemplo, Armando Rito opina que «la función debe regir el diseño, definiendo la forma» (15), y Hugo Corres dirá que «las ideas, el diseño conceptual y un profundo conocimiento del comportamiento estructural son las bases de todo gran proyecto» (16): Rito parece subordinar el diseño a la función, y Corres parece obviar la importancia del entorno paisajístico. Ambos, no obstante, han diseñado magníficos puentes.

Esta idea de la supremacía de «lo resistente» ha derivado en una determinada corriente estética ingenieril, asentada en la premisa de que la forma resistente óptima desde el punto de vista económico llevará a una solución estética igualmente válida. Según el profesor Billington esta corriente gobernó la estética ingenieril en los años posteriores a la Segunda Guerra Mundial debido a la necesidad de reconstruir las vías de comunicación con rapidez, y fue la causante de que muchos ingenieros perdieran interés en todo aquello que no estuviera

Si bien se tiene conocimiento de éste desde el siglo XIV, el puente de la imagen es ejemplo de diversas estructuras similares que jalonan la isla británica desde la época prehistórica. 
relacionado con la economía de medios (17). Creemos que los resultados de esta corriente estética son válidos e interesantes cuando el proyectista presenta dotes creativas.

En nuestra opinión, el proyecto de un puente está condicionado por numerosos parámetros (ya aludidos), siendo el resistente uno más. Un puente, hasta el más pequeño y común, siempre tendrá que enfrentarse a la cuestión ambiental y visual, y precisamente la sistemática desatención a estos aspectos es lo que lleva -pensamos- a que entre la sociedad y la ingeniería se establezca una barrera de difícil superación que lleva a considerar la obra civil como una suerte de no lugares paisajísticos insertados en el territorio -como en toda generalización, hay excepciones-. Deducimos entonces que no cabe hablar de utilidad primaria y cuestiones añadidas en términos secundarios: pensamos que si un proyecto no resuelve todos los problemas que citábamos antes, éste pierde su validez en tanto que proyecto. Así, centrando la cuestión en un tipo de puentes común en el territorio, no creemos en la sistemática repetición de modelos de pasos superiores en las carreteras, pues como indica Manterola el puente se convierte en un cliché y en un objeto casi industrial (18): el paisaje manchego, por ejemplo, poco tiene que ver con el de los Pirineos, y pese a ello los tipos constructivos realizados son similares. La industrialización de los «artefactos» construidos en el paisaje puede provocar la industrialización de la mirada y la pérdida del componente estético que subyace en la propia entidad humana. En este sentido, tal y como indican Muller y Dinis, los puentes modulares están haciéndose con un elevado porcentaje del mercado, y esta tendencia es constante y ascendente (19). La prefabricación, útil sin duda desde el punto de vista económico y del aseguramiento de la calidad en obra, introduce en ocasiones una inflexibilidad en el diseño de la que el ingeniero debería huir. La técnica del prefabricado debe estar supeditada a la voluntad del ingeniero, que podrá aprovecharse de las ventajas de la prefabricación y la modularización diseñando él mismo piezas «prefabricables» para su propio proyecto. Así ocurre con algunos pasos superiores del tramo Luko-Guipúzcoa de la Autopista VitoriaEibar (Juan J. Arenas, 2001), que logran distinguirse de los pasos convencionales merced al empleo de formas curvas y detalles en los acabados (Figura 4), como también ocurre con los pasos superiores de Carlos Fernández Casado dispuesto en la Autovía de La Coruña, en Madrid, destacados por Javier Rui-Wamba por su originalidad (20).

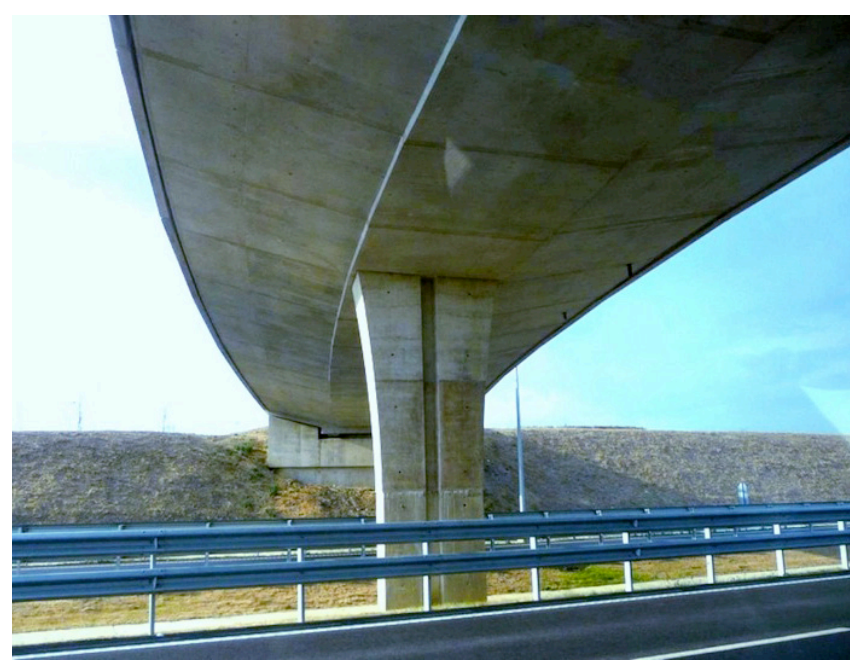

Figura 4. Uno de los pasos superiores en la autopista AP-1, Vitoria-Eibar.
En su fantástico libro, el proyectista Frederick Gottemoeller -autor del Puente Wilson (Washington, 2009), cuyas formas modernas dialogan paisajísticamente con los puentes históricos existentes en el Potomac (Figura 5) - desarrolla un argumento que termina por responsabilizar a los ingenieros sobre el escaso aprecio que la sociedad muestra hacia los puentes. Según él, «todos los puentes tienen un impacto estético; la sociedad está capacitada para apreciar la belleza de un puente del mismo modo que lo está para apreciar las bondades de una pintura o de un concierto; el ingeniero debe asumir toda la responsabilidad derivada del impacto estético de su puente; la capacidad estética es una habilidad que puede ser adquirida y desarrollada por los ingenieros como cualquiera de sus otras capacidades; los ingenieros deben atender la apariencia visual de su obra en el mismo plano de importancia que los aspectos técnicos y financieros» (21). Pero, como reconoce Jörg Schlaich, «la calidad general de muchos de nuestros puentes deja mucho que desear [...]. Con justificado orgullo, los ingenieros estructurales anuncian cada año el establecimiento de nuevos récords [...]. Sin embargo, nuestra vida diaria, nuestro entorno inmediato y el medio ambiente no se rigen por estas estructuras singulares espectaculares [...] sino por puentes, edificios, fábricas, chimeneas, muros, etc. de tamaño reducido y medio. Observando la mayoría de ellos [...] debemos admitir una falta considerable de calidad general. Con demasiada frecuencia son pobres, raros y grises, y no están en armonía con su entorno urbano o natural» (22). El propio Schlaich, en otro momento, se pregunta que «si todos los ingenieros poseen el conocimiento necesario para concebir un puente, y si la mayor parte de los humanos, desde su nacimiento, están dotados con algún tipo de intuición o creatividad, ¿̇por qué entonces hay tantos puentes miserables y tan pocos puentes brillantes?» (23). Prosigue el ingeniero indicando que «aunque otros productos de la creatividad humana como los coches, los muebles e incluso las casas unifamiliares son, en comparación con los puentes, sólo efímeros, existen expertos dedicados únicamente al diseño en cada una de las profesiones dedicadas a tales fines. En los puentes eso no ocurre. La enseñanza sobre el diseño de puentes en nuestras universidades todavía se limita al análisis y dimensionamiento, saltándose el diseño conceptual que determina la forma y que debe preceder al análisis dimensional para condicionarlo desde el primer momento» (24). La pasarela de Sassnitz (Alemania), firmada por Schlaich junto a Rudolf Bergermann en 2007, es ejemplo de cómo hallar relaciones formales entre el puente y su entorno territorial: su planta ondulada nos relaciona con el mar y su oleaje, con el dique y con las formas costeras (Figura 6).

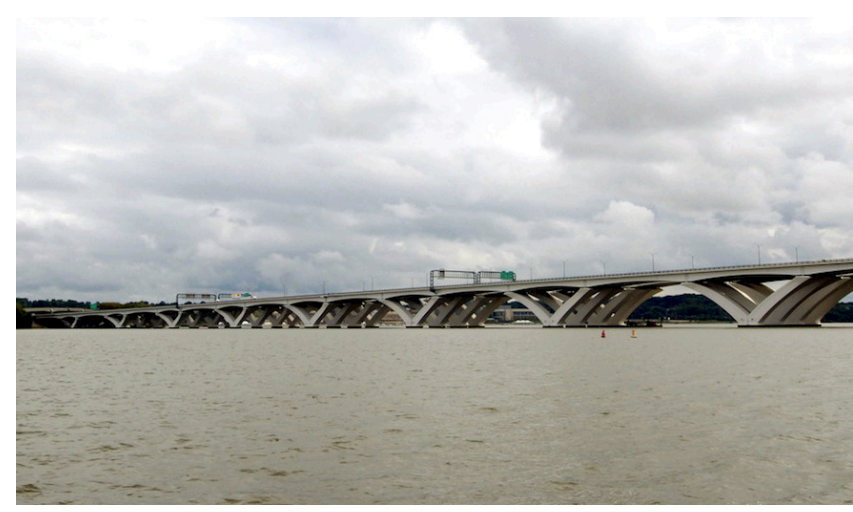

Figura 5. Puente Wilson, en Washington. 


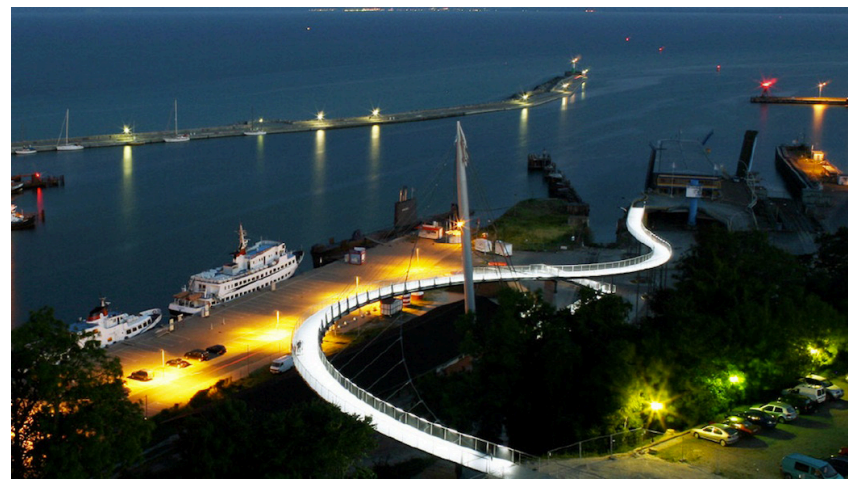

Figura 6. Pasarela de Sassnitz.

En parecidos términos se manifiesta Catharina Ottander, del Ministerio de Fomento sueco, al escribir en un libro de elocuente título que «puentes y carreteras deberían parecer y sentirse como una parte natural del territorio existente. Debemos recordar que los puentes que construimos hoy estarán aún en uso durante el próximo siglo. Por eso tenemos que invertir en calidad, estética y medio ambiente» (25). Del mismo modo, Steinman escribe que «los puentes son un índice de civilización y progreso, y no se debe diseñar ningún puente importante sin consagrar ideas y esfuerzo para conseguir belleza» (26). Y Fernández Casado también pondrá en relación la mirada con el puente, pues advierte, en su manual de cálculo de puentes, que estas estructuras, a diferencia de otras obras de ingeniería que son ocultas, «al destacarse en el aire han de ser necesariamente o airosas o torpes. [...] $\mathrm{Su}$ forma material, corpórea, quedará expuesta al público [...], sujeta a la crítica de sus contempladores» (27). Dirá también que «el volumen del puente, con su frontalidad privilegiada y en la mayor parte de los casos distribuido en simetría, se enfrenta con nuestra corporeidad. El equilibrio de esfuerzos que en todo momento ha de asegurar su estructura, armoniza con nuestra condición de seres vigilantes para mantenernos en pie» (28). Fernández Casado parece indicar la necesidad de prestar atención a la estética del puente, a diferencia de las obras ingenieriles de estructura no expuesta, y a la relación estética que aparece entre él y el propio observador.

Por último, cabe señalar el peligro de la excesiva ponderación de «lo espectacular». En los últimos tiempos han comenzado a proliferar determinados puentes y pasarelas en contextos urbanos que buscan significarse en el territorio mediante el empleo de tipologías complejas y materiales poco comunes -y usualmente caros-. Del mismo modo que la construcción del museo Guggenheim de Bilbao provocó el llamado «efecto Guggenheim», posiblemente la construcción del puente Lusitania de Mérida (Santiago Calatrava, 1991), convertido en símbolo de la entrada de la capital extremeña en una nueva etapa histórica -el «efecto Lusitania»- e incluido en numerosas publicaciones monográficas, fuera el detonante de la búsqueda del puente-símbolo por parte de distintos Ayuntamientos. El resultado de estos puentes que buscan deliberadamente contentar a la ciudadanía ha sido desigual, y como ya indicábamos al principio del epígrafe, está condicionada por la creatividad y habilidad del proyectista. Creemos además que la sociedad en su conjunto tampoco valora la ingeniería-espectáculo, salvo en unos pocos casos concretos, pues un puente notablemente ostentoso que no llega a convertirse en símbolo urbano capaz de atraer miradas foráneas puede ser entendido como un derroche, máxime en tiempos de crisis. Las inversiones en puentes-símbolo, por ello, deberían ser operaciones cuidadosamente medidas en términos presupuestarios, analizando los posibles retornos de la inversión.

Un ejemplo de operación exitosa es el Gateshead Millennium Bridge (Gifford, Wilkinson Eyre, 2002) de Newcastle; en este caso las bases del concurso indicaban que la nueva estructura debía constituir tanto un símbolo de la ciudad como un complemento al resto de puentes erigidos en el río Tyne y un ejemplo de innovación ingenieril «como también lo fueron sus predecesores en el mismo río y en la misma ciudad cuando fueron construidos", entre otras premisas. Es decir, que junto a la idea de símbolo se consideraba también la necesidad de que la pasarela se integrase en su entorno y dialogara con las estructuras existentes. El Gateshead Millennium Bridge ha sido considerado como un ejemplo a seguir para el resto de los ingenieros (29).

\section{EL PUENTE COMO PROBLEMA PAISAJÍSTICO}

Hemos visto entonces cómo Manterola, Gottemoeller, Schlaich, Ottander o Fernández Casado, junto a otros como Calatrava (30) o Elliott (31), alertan de la ausencia del interés del ciudadano medio hacia los puentes; algunos de ellos llegan a responsabilizar de este desapego a los propios proyectistas, que obvian o consideran secundaria la componente estética de la obra civil frente a otros parámetros. Si pensamos en términos mercantiles, es obvio que el interesado en vender un producto es quien ha de mostrar las virtudes de dicho producto a un potencial comprador. Los ingenieros americanos Barker y Puckett opinan en el mismo sentido, y critican que algunos ingenieros carezcan de sensibilidad estética, al no ir más allá de una serie de fórmulas que determinen, a partir de unas cargas, del tráfico que han de soportar y de las características geológicas del lugar, cómo ha de diseñarse un puente (32).

Realmente, cuando hablamos de la estética de elementos dispuestos en el territorio estamos hablando de paisaje (que definimos como una construcción mental donde intervienen cuatro elementos: un territorio, un observador, un medio por el que se produce dicha observación y unos vínculos existentes entre el territorio y el observador que influyen en la acción de observar), por lo que el problema estético de la obra ingenieril es, hablando con propiedad, un problema paisajístico. Para abordar el encaje de su obra en el territorio, el proyectista ha de jugar con diversas variables: proporciones, simetría, ritmo, función, integración, color y textura de materiales, carácter, contraste entre forma y masa, estabilidad visual o compatibilidad histórica con el entorno. La solución, por tanto, no será única, e incluso el carácter de la solución adoptada posiblemente provocará opiniones divergentes en opinadores y analistas. Si tomamos por ejemplo el neoyorquino puente de Bayona sobre el estrecho del Kill van Kull, podremos ver críticas diversas en lo referente a los estribos del arco -dicho puente guarda similitudes con el puente del puerto de Sydney y con el Hell Gate de Nueva York (33), pero a diferencia de ellos carece de elementos de sillería en dichos estribos (Figura 7)-. Así, el historiador americano Carl W. Condit criticaba el diseño general del puente, alabando al tiempo el dinamismo del arco: Condit se lamentaba de que no se hubiese llevado a cabo la idea del arquitecto Cass Gilbert, quien propuso revestir con granito las torres que enmarcan al arco, y que resultan estructuralmente necesarias (34). Frente a la opinión de Coldit, sin embargo, encontramos la de Javier Manterola, que muestra su aprecio hacia el puente tal y como 
está, pues «la fuerza, la potencia sin concesiones para salvar su formidable luz de $503 \mathrm{~m}$ aplasta todo intento ornamental, que no puede ni insinuarse» (35). Y es que la cuestión de cómo afrontar las pilas de los puentes metálicos ante la posibilidad de utilizar el metal desprovisto de ornato o la sillería, más dada a lo monumental, perduró hasta la aparición del hormigón.
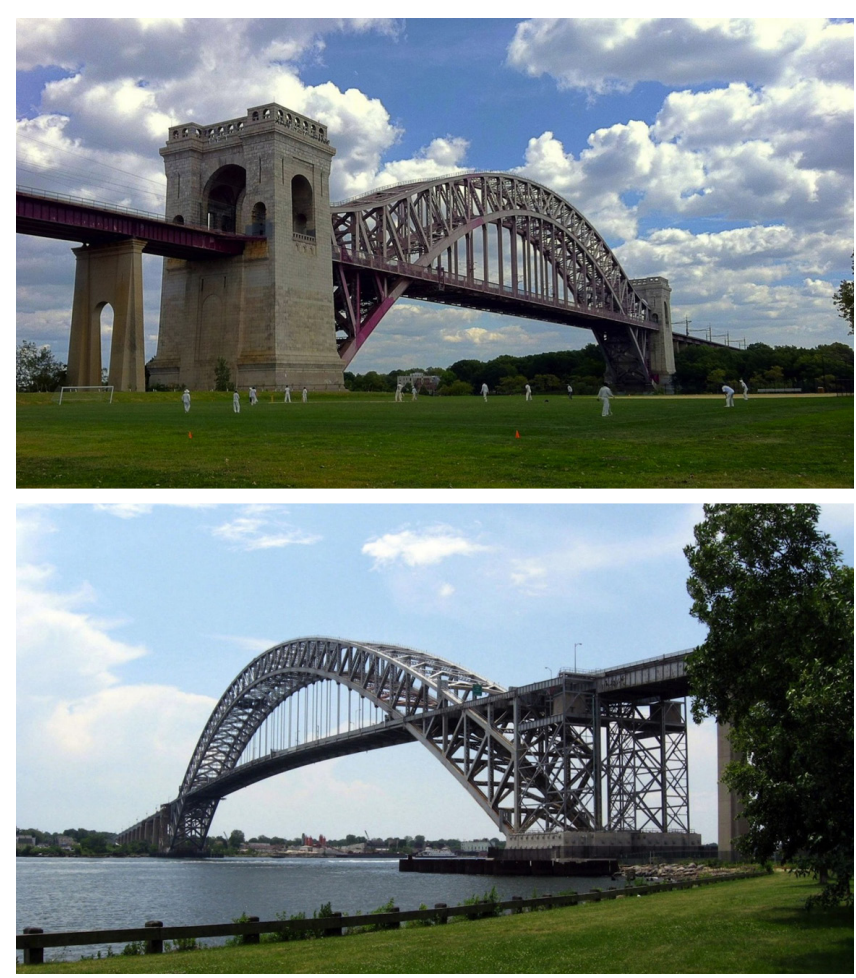

Figura 7. Dos modos de resolver las pilas en puentes no muy distantes entre sí: el Hell Gate (Gustav Lindenthal, 1916) y el puente de Bayona (Othmar Ammann, Allston Dana, Cass Gilbert, 1931).

\section{CUATRO VÍAS DE ACERCAMIENTO DE LA SOCIEDAD HACIA LOS PUENTES}

El escaso interés paisajístico que los puentes despiertan en la población se ciñe, sin embargo, a la vía de la observación directa en la vida cotidiana (es decir, a uno de los «modos» posibles de observar el territorio); de hecho, sí detectamos un total de cuatro vías a través de las cuales el ciudadano medio va a relacionarse con los puentes, apreciando además determinadas virtudes físicas, visuales o culturales en ellos.

Una primera viene dada por el elevado número de representaciones y reproducciones artísticas de diversos puentes, sean éstos reales o ficcionales. Hablamos entonces de otros «modos» de observar el territorio, más allá de esa observación directa anteriormente aludida. La importancia del puente en la pintura ha sido estudiado por diversos investigadores como John Sweetman (36), David McFetrich (37), o Ana Vázquez de la Cueva (38), que demuestran su relevancia en tanto que elementos visuales de indudable capacidad sugestiva y metafórica. Del mismo modo, y junto a trabajos de vocación generalista como Chale Nafus (39) o Valentín Alejándrez et al. (40), encontramos investigaciones sobre las relaciones existentes entre el cine y los puentes (41), demostrando la relevancia del puente en el lenguaje cinematográfico de un autor (42) o las capacidades escenográficas de un puente determinado (43). En con- junto, estos textos demuestran cómo la representación del puente en un soporte artístico de notable difusión sí acerca estas obras civiles al conjunto de la sociedad, elevando su consideración en el imaginario colectivo. También la televisión logra potenciar este efecto, creando iconos urbanos -¿qué imagen, por ejemplo, procurará tener tras de sí un corresponsal que retransmite una noticia desde San Francisco, Londres o Florencia?-.

La segunda vía de aproximación está relacionada con la dificultad que se presupone al hecho de proyectar y posteriormente construir un puente. Como ejemplo de esa idea, podemos recordar cómo en la antigua Roma los puentes eran concebidos por una serie de sacerdotes de una determinada institución religiosa; dicha institución recibía el nombre de Collegium Pontifices y su cabeza era el Pontifex Maximus, que podríamos traducir como «el más grande hacedor de puentes» (44). La existencia de los Pontifex romanos y su consideración de sacerdotes reflejan la creencia, por parte del vulgo, de que las actividades de concebir y materializar un puente estaban vinculadas a la adquisición de algún tipo de conocimiento extraordinario y cercano a la gnosis. Ya en época medieval, apuntamos la tradición existente en distintos lugares de Europa que lleva a atribuir al Diablo la construcción de puentes especialmente complejos (45), como los representados en la Figura 8; estas leyendas muestran la creencia, por parte de la sociedad medieval, de que la construcción del puente era una actividad prodigiosa, lo cual no resulta extraño: al comparar las anchuras de las naves de las catedrales góticas y los arcos construidos para los puentes, vemos cómo los guarismos delatan significativas diferencias: frente a los 22,98 $\mathrm{m}$ de anchura de la catedral de Gerona -considerada la nave gótica más ancha del mundo-, encontramos cómo el puente del Diablo de Martorell llega a los 37,3 m y el de Ceret de nombre similar, en Francia, alcanza los 45,45 m. Aun no dudando de la magnificencia de las catedrales góticas (cuya compleja disposición de pesos y contrapesos no es inteligible tampoco con facilidad), cabe sorprenderse frente a la liviandad de una sencilla línea de piedras capaz de discurrir por el vacío sin más sostén que la forma del conjunto y la acertada disposición de las juntas entre la piedra anterior y la siguiente. Ambos ejemplos, relacionados con la época romana y medieval, demuestran el impacto que causan en la sociedad los puentes-récord y los puentes de construcción compleja. Cuando Manterola reflexiona sobre la relación puente-sociedad apela también a esta segunda vía. Así, el navarro escribe que «para un ciudadano normal, el significado (de un puente) puede oscilar desde una simple impresión neutral, como algo que tiene que existir para que puedan realizarse intercambios físicos suficientemente rápidos y seguros, a una impresión favorable cuando su presencia se convierte en asombro ante la dimensión, importancia y contundencia del puente [...]; aunque difícilmente se sale de ese "estar asombrado"» (46). Añadimos nosotros que la valoración de la obra pública atendiendo exclusivamente a estos parámetros presenta sin embargo un inconveniente: la caducidad de los mismos. Conforme la técnica va permitiendo mayores logros dimensionales, los antiguos puentes-récord pierden la vitola que los adornaba, y el citius, altius, fortius constructivo se traslada a otra estructura. Por ello, no consideramos que el mérito del récord pueda ser tenido como una virtud de orden paisajística en lo que se refiere a los puentes. 

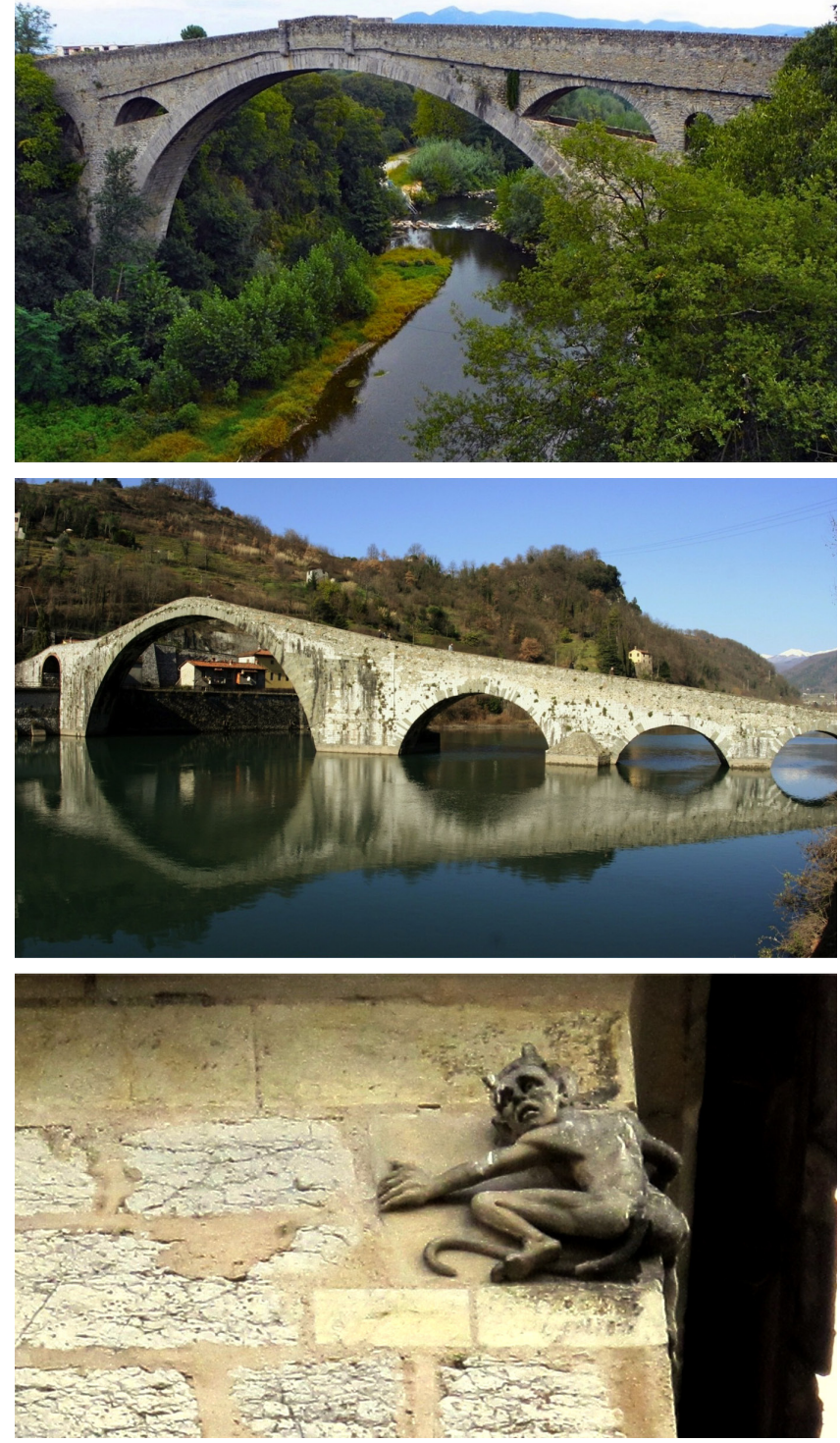

Figura 8. Puentes del Diablo de Ceret (1321-1341) y de Borgo a Mozzano en Italia (1080-1100), junto a un detalle del puente de Valentré (Paul Gout, 1350).

La tercera vía de aproximación entre la sociedad y los puentes nos remite al propio significado cultural que éstos representan. Así, en la cultura islámica el puente es el lugar donde seremos juzgados tras la muerte, precisamente por ser un lugar de transición entre la tierra y el cielo (47); por otra parte, y por mor de la oficialización del cristianismo como religión del Imperio Romano, el citado cargo de Pontifex Maximus sería asumido por el máximo representante de la nueva religión en la ciudad, el Obispo de Roma (48). Dado que el título no llevaba aparejado la adquisición inmediata de conocimientos estructurales, los puentes construidos por el Obispo romano -o Papa- serían metafóricos. Más allá de estos apuntes que evidencian la fuerte vinculación del concepto puente con el sustrato cultural de dos religiones, parece evidente que, como dice Arenas, los puentes constituyen unos elementos indispensables para el desarrollo de la civilización y la cultura (49), y su existencia pone de manifiesto la antropización de un territorio -Scott Corbett, con fino humor inglés, inicia su libro Bridges escribiendo que «en algún sitio en el mundo debe haber gente que nunca ha cruzado un puente. Tiene que haber alguien en el Sahara, por ejemplo» (50)-; el propio Arenas aportará un ejemplo descriptivo de esta idea: «el há- bitat natural de la vida humana es el valle, donde hay tierra cultivable, donde el clima es más suave y donde hay ríos de los que tomar el agua necesaria. Pero esos cauces, auténtica base de la vida, suponen también una fuerte limitación para su desarrollo al coartar, de modo a veces imperioso, la movilidad de los hombres. El puente viene a ser así el elemento clave superador de la antítesis de apoyo a la vida y de freno a la civilización que los grandes ríos representan» (49) -algo también apuntado por Corbett (50)-. Además, y a partir de ahí, «el puente ha seguido simbolizando el punto de máxima tensión en el esfuerzo del hombre por humanizar la naturaleza» (51), e incluso en la actualidad es símbolo de un grupo social que tendrá en común el pago de tasas a una misma administración: la obra pública, sufragada por la colectividad con sus impuestos, adquirirá «el valor de muestra, simbólica y real al tiempo, del correcto funcionamiento democrático de una sociedad» (52). Y si bien este pensamiento dista bastante de lo que entiende el ciudadano por el cobro de impuestos, sí reconocemos que en puridad el puente es símbolo de un logro grupal que va a permitir una mayor facilidad de vida, y que surge en el paisaje como índice del avance tecnológico de la sociedad y como muestra de la pujanza económica de un determinado país, región o ciudad. Y así como el dictador Salazar bautizó con su nombre el puente de Lisboa -símbolo del renacimiento económico portugués bajo su mandato- para situar ficticiamente a su país a la altura tecnológica de las principales potencias de la época, también Cádiz ha pretendido celebrar el Bicentenario de la Constitución de 1812 con la inauguración de un puente atirantado que cruce la Bahía.

La última vía de relación entre sociedad y puente nos relaciona con el interés que puede suscitar el hecho constructivo puente en determinadas personalidades de elevado prestigio intelectual, cuya preeminencia en la esfera humanística, filosófica o científica sea tal que sus opiniones y escritos logren influir en todo su ámbito, e incluso más allá del mismo. En este sentido, y a modo de ejemplo, cabe referirse a la conferencia que impartió el filósofo Martin Heidegger en 1951 en la Escuela de Arquitectura de Darmstadt (Alemania), y que llevaba por título Construir, habitar, pensar. Heidegger por esa época era una autoridad filosófica consumada cuya relevancia abarcaba los más diversos campos, y fue invitado para exponer su particular visión acerca del fenómeno de la construcción. El pensador relacionaba la construcción con la acción de habitar el territorio, e introducía el concepto de Cuaterna -o Cuaternidad, según la fuente que consultemos (53) (54) - como unidad que hay que preservar de cara a la consecución del verdadero habitar. La Cuaterna sagrada consta de cuatro elementos: Tierra, Cielo, los hombres mortales y los enviados de la Divinidad; en ella todas las partes son una unidad original, y cada una de ellas sólo puede concebirse a partir de las tres restantes. Heidegger propone el puente como elemento constructivo que permite mostrar la verdadera unidad de Cielo, Tierra, Hombres y Dioses (o lo Divino, o lo Supranatural, o lo Trascendental, o lo Ideológico), y a partir de ahí escribe una serie de consideraciones sobre la naturaleza del mismo como construcción que permite simultanear la ideas de la ligazón con la del movimiento y del paso de un estado a otro. Así, dirá que «es pasando por el puente como aparecen las orillas en tanto que orillas. El puente es propiamente lo que deja que una yazga frente a la otra. [...] El puente, con las orillas, lleva a la corriente las dos extensiones de paisaje que se encuentran detrás de estas orillas. Lleva la corriente, las orillas y la tierra a una vecindad recíproca. El puente coliga la tierra como paisaje en 
torno a la corriente» (54). También indicará que «el puente, si es un auténtico puente, no es nunca primero puente sin más y luego un símbolo» (55). Del texto de Heidegger extraemos tres conclusiones: de una parte, que expresa con notable precisión la preeminencia del puente en tanto que «hecho paisajístico» (no en vano, podemos definir el paisaje como un concepto de naturaleza cuatérnica donde la Tierra es el territorio; el Cielo, los vínculos que el observador logra crear mentalmente con el lugar y los elementos observados; los hombres, el observador; los enviados de la Divinidad, el medio empleado para relacionar los tres elementos anteriores). De otra, que la elección del puente como epítome de la actividad constructiva del hombre en el territorio supone un hecho de enorme magnitud que eleva "por asociación» al puente como construcción emblemática desde el punto de vista de la filosofía, y cuya trascendencia no podrá ser ya ignorada. Por último, que cuando indica que «el puente no es primero puente sin más y luego un símbolo» está aludiendo a lo que nosotros sugeríamos unas páginas antes: que el puente no presenta una utilidad primaria y cuestiones añadidas en términos secundarios, y que si un proyecto no resuelve todos los problemas que citábamos antes, el proyecto no sirve. Finalmente, y ya relacionado con la idea de la cuarta vía de aproximación puente-sociedad, observamos que cuando Heidegger utiliza el puente como metáfora del habitar o como símbolo de la Cuaterna está vinculando el campo filosófico con el campo ingenieril, provocando el interés de los ingenieros por Heidegger -Heidegger ha sido citado en numerosas ocasiones por ingenieros y urbanistas como Arenas (53), Frampton (56), Lanza (57) o Fernández Casado (58) - y el de los filósofos por la ingeniería civil como muestra la especial relación entre Carlos Fernández Casado y Xabier Zubiri (59) - dada la influencia de Heidegger en la filosofía contemporánea (60).

\section{ALGUNAS CONCLUSIONES}

El texto ha realizado un recorrido en torno a las relaciones que se establecen entre los puentes y los usuarios visuales del mismo, y de un modo más genérico, entre los proyectistas de puentes y el conjunto de la sociedad. A lo largo del mismo se han ido distinguiendo una serie de ideas que extraemos, sintetizamos y ordenamos a modo de conclusiones de la reflexión realizada:

1. Los puentes presentan cualidades desde diversos puntos de vista: como ideal constructivo, como símbolo de toda una sociedad, como logro tecnológico con el que vencer un obstáculo, como monumento, como superador de fronteras o como herramienta económica y comercial.

2. El puente es el hecho icónico de la ingeniería civil.

3. En el puente se dan cita los principales problemas a los que el ingeniero se enfrenta cuando proyecta una obra. Entre ellos se encuentra la integración de la obra en el territorio donde se ubica.

4. La integración paisajística de un puente en el territorio depende de diversas variables, y éstas presentarán un amplio abanico de soluciones posibles y válidas.

5. La sociedad no siempre aprecia las virtudes estéticas que un puente lúcido presenta por sí sola; más allá de una posible falta de formación ciudadana con respecto a la ingeniería, el problema puede venir dado por la insuficiente atención prestada por parte de algunos proyectistas al problema paisajístico que subyace en el hecho de disponer un elemento en el territorio; prueba de ello es el elevado número de puentes mediocres construidos, que además ocultan la valía de los que son brillantes.

6. El puente ha sido empleado como símbolo del éxito y pujanza de una colectividad o de un poder.

7. Los acercamientos de la sociedad hacia los puentes han venido dados por cuatro vías: por elevación, al presentarlos reproducidos mediante algún tipo de arte asentado en el espacio de lo poético y de lo estético, como la pintura o el cine; por su complejidad, tal y como atestigua la común asociación entre puentes y hechos sobrenaturales o religiosos; por su capacidad metafórica, al representar culturalmente tanto la vinculación entre el cielo y la tierra como la humanización de la naturaleza; por la apreciación que del mismo hacen personas intelectualmente relevantes, como demuestra el hecho de que Heidegger lo emplee como elemento que ejemplifica su Cuaterna (la unión de cielo, tierra, lo humano y lo divino).

Estos siete puntos nos llevan a una última reflexión: los puentes son elementos que carecen de connotaciones negativas en la sociedad (incluso hemos visto cómo son empleados en tanto que metáforas de relaciones de tipo trascendental); ante la ausencia un sustrato cultural que imponga una visión negativa de los puentes al imaginario colectivo, cabe deducir que su consideración de no-lugares paisajísticos es responsabilidad de los proyectistas. Sólo ellos podrán revertir dicha situación. Y esto sólo tendrá lugar cuando se considere como fundamental, durante el desarrollo conceptual del proyecto, el problema paisajístico latente en todo elemento dispuesto sobre el territorio. Algo que, por otra parte, han hecho numerosos técnicos a lo largo de la historia.

\section{REFERENCIAS}

(1) Monterroso, A. (1959). El dinosaurio. Obras completas (y otros cuentos). México: UNAM.

(2) Steinmann, D. B., Watson, S. R. (1979). Puentes y sus constructores, pp. 15-16. Madrid: Turner.

(3) Galliazzo, V. (1994). I ponti romani, pp. 556-565. Treviso: Canova.

(4) Steinmann, D. B., Watson, S. R. (1979). Puentes y sus constructores, p. 59. Madrid: Turner.

(5) Hernández-Fernández, S. (2009). Puentes de España. Testigos de nuestro pasado, p. 9 (Prólogo). Barcelona: Lunwerg.

(6) Manterola, J. (2010). La obra de ingeniería como obra de arte, pp. 28-110. Pamplona: Laetoli.

(7) Siegrist-Ridruejo, G. (2006). Arco de Almonte. Autovía de la Plata. Tramo Hinojal Cáceres. Hormigón y Acero, 240: 5-29.

(8) Rossi, A. (2007). La arquitectura de la ciudad, p. 188. Barcelona: Gustavo Gili.

(9) Martínez-Calzón, J. (2006). Puentes, estructuras, actitudes, p. 26. Madrid: Turner.

(10) Abercrombie, P. (1975). The aesthetic aspect of civil engineering design; a record of six lectures delivered at the Institution, pp. 71-81. Londres: The Institutution of Civil Engineers.

(11) Steinmann, D. B., Watson, S. R. (1979). Puentes y sus constructores, p. 391. Madrid: Turner. 
(12) Aguiló, M. (2010). Forma y tipo en el arte de construir puentes, p. 19. Madrid: Abada.

(13) Billington, D. P. (1977). Structural art and Robert Maillart. Architectural Science Review, 20(2): 44-52, doi: http:// dx.doi.org/10.1080/00038628.1977.9697236.

(14) Barker, R. M., Puckett, J. A. (2007). Design of highway bridges. An LRFD approach, p. 1. Hoboken: John Wiley \& Sons, Inc.

(15) Rito, A. (200o). Estética y diseño en puentes. En Tendencias en el Diseño de Puentes, (p. 277). Barcelona: Grupo español del IABSE.

(16) Corres-Peiretti, H. (200o). Diseño conceptual: un procedimiento ingenieril para obtener soluciones formalmente interesantes. En Tendencias en el Diseño de Puentes, (p. 165). Barcelona: Grupo español del IABSE.

(17) Billington, D.P. (2006). Teaching ethics in engineering education through historical analysis. Science and Engineering Ethics, 12(1): 214, doi: http://dx.doi.org/10.1007/s11948-006-0021-z.

(18) Manterola, J. (2010). La obra de ingeniería como obra de arte, p. 103. Pamplona: Laetoli.

(19) Muller, J., Dinis, A. (200o). Sistemas modulares en puentes. En Tendencias en el Diseño de Puentes, (p. 47). Barcelona: Grupo español del IABSE.

(20) Rui-Wamba Martija, J. (2000). Puentes españoles de hogaño. En Tendencias en el Diseño de Puentes, (p. 395). Barcelona: Grupo español del IABSE.

(21) Gottemoeller, F. (2004). Bridgescape. The art of Designing Bridges, pp. 5-11. Hoboken: John Willey \& Sons, Inc.

(22) Schlaich, J. (2000). Variedad en el diseño de puentes. En Tendencias en el Diseño de Puentes, (p. 69). Barcelona: Grupo español del IABSE.

(23) Schlaich, J. (2004). Foreword to the second edition. En Bridgescape. The art of Designing Bridges (p. VII). Hoboken: John Willey \& Sons, Inc.

(24) Schlaich, J. (2004). Foreword to the second edition. En Bridgescape. The art of Designing Bridges (p. VI). Hoboken: John Willey \& Sons, Inc.

(25) Ottander, C. (1997). A work of art: The high coast project with its 35 bridges to the future, p. 47. Vägverket: Page One Publishing.

(26) Steinman, D. B., Watson, S. R. (1979). Puentes y sus constructores, p. 387. Madrid: Turner.

(27) Fernández-Casado, C. (1961). Puentes de hormigón armado pretensado. Generalidades y Cálculo, p. 1. Madrid: Dossat.

(28) Fernández-Casado, C. (1961). Puentes de hormigón armado pretensado. Generalidades y Cálculo, p. 5. Madrid: Dossat.

(29) Johnson, J., Curran, P. (2003). Gateshead Millenium Bridge -an eye-opener for engineering. Proceedings if ICE, Civil Engineering, 156:19.

(30) Calatrava, S. (2003). Conversaciones con estudiantes, p. 6o. Barcelona: Gustavo Gili.

(31) Elliott, A.L. (1983). Esthetic development of California's bridges. Journal of Structural Engineering, 109(9): 2162, doi: http://dx.doi.org/10.1061/(ASCE)0733-9445(1983)109:9(2159).

(32) Barker, R. M., Puckett, J. A. (2007). Design of highway bridges. An LRFD approach, p. 45. Hoboken: John Wiley \& Sons, Inc.

(33) Thrall, A. P., Billington, D. P. (2008). Bayonne Bridge: the work of Othmar Ammann, Master Builder. Journal of Bridge Engineering, 13 (6): 635-643, doi: http://dx.doi.org/10.1061/(ASCE)1084-0702(2008)13:6(635).

(34) Condit, C. W. (1961). American building art: the twentieth century, p. 235. Nueva York: Oxford University Press.

(35) Manterola, J. (2010). La obra de ingeniería como obra de arte, p. 94. Pamplona: Laetoli.

(36) Sweetman, J. (1999). The artist and the bridge: 1700-1920. Londres: Aldershot.

(37) McFetrich, D. (2006). Spanning the river: artists'views of Thames bridges. Londres: Guildhall Art Gallery.

(38) Vázquez-de la Cueva, A. (2006). Ingeniería y pintura. Ingeniería y territorio. De la ingeniería y las otras artes, I(78): $50-51$.

(39) Nafus, Ch. (2003). Celluloid Connections: The Bridge in Cinema. Austin: Historic Bridge Foundation.

(40) Alejándrez, V. J., Magallón, G., Bisbal I., Pereña, R. M. (2005). La obra civil y el cine. Una pareja de película. Madrid: Cinter.

(41) Plasencia-Lozano, P. (2012). El paisaje de los puentes urbanos. La mirada del cine. El New hollywood y Woody Allen (Tesis Doctoral no publicada). Cáceres: Universidad de Extremadura.

(42) Plasencia-Lozano, P. (2010). Los puentes como argumento estético en la trilogía del hampa de Brian de Palma. NorbaArte, 30: 227-246.

(43) Plasencia-Lozano, P. (2011). El puente de Brooklyn y el cine. Análisis fílmico-estético de la estructura. En Segundo Congreso Internacional de Historia y Cine, (pp. 1095-1113). Madrid: T\&B Ediciones, doi: http://dx.doi.org/10016/11403.

(44) Steinman, D. B., Watson, S. R. (1979). Puentes y sus constructores, p. 59. Madrid: Turner.

(45) Martínez-Reche, A. (2001). Puentes y diablos, o la demonización del saber técnico. OP Ingeniería y territorio, Ingeniería e Historia, 56: 90-95.

(46) Manterola, J. (2010). La obra de ingeniería como obra de arte, p. 113. Pamplona: Laetoli.

(47) Roig, J. (1996). Nuevos puentes, p. 12. Barcelona: Gustavo Gili.

(48) Steinman, D. B., Watson, S. R. (1979). Puentes y sus constructores, p. 6o. Madrid: Turner.

(49) Arenas, J. J. (2002). Caminos en el aire. Los puentes, p. 25. Madrid: Colegio de Ingenieros de Caminos, Canales y Puertos.

(50) Corbett, S. (1978). Bridges, p. 1. Nueva York: Four Wind Press.

(51) Arenas, J. J. (2002). Caminos en el aire. Los puentes, p. 33. Madrid: Colegio de Ingenieros de Caminos, Canales y Puertos.

(52) Arenas, J. J. (2002). Caminos en el aire. Los puentes, p. 509. Madrid: Colegio de Ingenieros de Caminos, Canales y Puertos. 
(53) Arenas, J. J., (2002). Caminos en el aire. Los puentes, p. 43. Madrid: Colegio de Ingenieros de Caminos, Canales y Puertos.

(54) Heidegger, M. (2001). Construir, habitar, pensar. En Conferencias y artículos, p. 112. Barcelona: Ediciones del Serbal.

(55) Heidegger, M. (2001). Construir, habitar, pensar. En Conferencias y artículos, p. 113. Barcelona: Ediciones del Serbal.

(56) Frampton, K. (1993). The architect as bridge builder. En Calatrava Bridges. p. 161. Zurich: Artemis.

(57) Lanza, C. (2007). Andamios para las ideas. Los lenguajes de la ingeniería, sus filosofías y apaños. Ingeniería y territorio. De la ingeniería y las otras artes, I(78): 24-35.

(58) Fernández-Casado, C. (1980). Historia del puente en España. Puentes Romanos, p. 26. Madrid: Colegio de Ingenieros de Caminos, Canales y Puertos.

(59) San Baldomero, J. M. (1992). Ingeniería y Filosofía. Carlos Fernández Casado y Xavier Zubiri. Logroño: Centro de Estudios riojanos-Gobierno de La Rioja.

(60) Lackey, D. (1999). What Are the Modern Classics? The Baruch Poll of Great Philosophy in the Twentieth Century. Philosophical Forum, 30(4): 329-346, doi: http://dx.doi.org/10.1111/oo31-806X.00022. 